\title{
Increased mechanical cost of walking in children with diplegia: The role of the passenger unit cannot be neglected
}

\author{
P. Van de Walle a,b,c,*, A. Hallemans ${ }^{\mathrm{a}, \mathrm{d}, \mathrm{f}}, \mathrm{S}$. Truijen ${ }^{\mathrm{a}, \mathrm{e}}, \mathrm{R}^{\mathrm{a}}$ Gosselink ${ }^{\mathrm{c}}$, L. Heyrman ${ }^{\mathrm{c}}$, \\ G. Molenaers ${ }^{\mathrm{b}, \mathrm{g}}$, K. Desloovere ${ }^{\mathrm{b}, \mathrm{c}}$

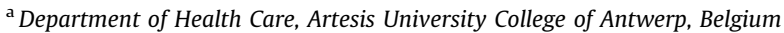 \\ ${ }^{\mathrm{b}}$ Clinical Motion Analysis Laboratory, CERM, University Hospital Pellenberg, Belgium \\ ${ }^{\mathrm{c}}$ Department of Rehabilitation Sciences, Faculty of Kinesiology and Rehabilitation Sciences, KU Leuven, Belgium \\ ${ }^{\mathrm{d}}$ Department of Biology, Functional Morphology, University of Antwerp, Belgium \\ ${ }^{\mathrm{e}}$ Department of Rehabilitation Sciences and Physiotherapy, Faculty of Medicine and Health Sciences, University of Antwerp, Belgium \\ ${ }^{\mathrm{f}}$ Department of Translational Neurosciences, Faculty of Medicine and Health Sciences, University of Antwerp, Belgium \\ ${ }^{\mathrm{g}}$ Department of Paediatric Orthopedics, Faculty of Medicine, KU Leuven, Belgium
}

\section{A R T I C L E I N F O}

\section{Article history:}

Received 19 March 2012

Received in revised form 30 May 2012

Accepted 30 May 2012

Available online 27 June 2012

\section{Keywords:}

Cerebral palsy

Gait

Mechanical energy

Upper body

\begin{abstract}
A B S T R A C T
Gait efficiency in children with cerebral palsy is decreased. To date, most research did not include the upper body as a separate functional unit when exploring these changes in gait efficiency. Since children with spastic diplegia often experience problems with trunk control, they could benefit from separate evaluation of the so-called 'passenger unit'. Therefore, the aim of the current study was to improve insights in the role of the passenger unit in decreased gait efficiency in children with diplegia. Mechanical cost of walking was investigated by calculating work by the integrated joint power approach in 18 children with diplegia and 25 age-related typical developing controls. The total mechanical work in children with diplegia was 1.5 times higher than in typical children. In children with diplegia work at the lower limbs was increased by $37 \%$ compared to typical children. Substantially higher increases, up to $222 \%$, were noted at the passenger unit. Trunk and head were the main contributors to the increased work of the passenger unit, but the role of the arms cannot be neglected.

Due to these disproportional increases in locomotor and passenger unit, the demands of the passenger unit in pathological gait can no longer be considered minor, as in typical gait. Therefore, the role of the passenger unit must be recognized in the decrease of gait efficiency in children with spastic diplegia and should be part of the evaluation of gait efficiency in clinical practice.
\end{abstract}

(c) 2012 Elsevier Ltd. All rights reserved.

\section{Introduction}

Cerebral palsy (CP) describes a group of permanent disorders of the development of movement and posture, causing activity limitation, that are attributed to non-progressive disturbances that occurred in the developing foetal or infant brain (Rosenbaum et al., 2007). The changes in motor capacities in children with CP are affecting their gait pattern and lead to a decrease in gait efficiency and thus functionality in daily living (Bennett et al., 2005; Gage, 1991; Kerr et al., 2008; Piccinini

\footnotetext{
* Corresponding author at: Clinical Motion Analysis Laboratory, University Hospital Pellenberg, Weligerveld 1, B-3212 Pellenberg, Belgium. Tel.: +32 4959256 27; fax: +32 495925627 .

E-mail address: vandewalle@artesis.be (P. Van de Walle).
} 
et al., 2007; van den Hecke et al., 2007). Adequate decision making in treating the gait problems in children with CP relies on a sound understanding of the pathological mechanisms causing gait abnormalities, such as decreased gait efficiency (Gage, 1991).

Gait efficiency can be assessed by the measurement of metabolic cost or by the estimation of mechanical work during walking. Where metabolic cost can only adopt the overall penalty of pathological gait, mechanical work can be assessed for different body parts at each instant of the gait cycle. This can reveal invaluable information on causes of increased energy expenditure. Mechanical work can be calculated by different approaches, all based on the estimation of work from kinematics and/or kinetics, as there are: (1) calculation of external work, (2) sum of segmental energies and (3) integration of joint powers (Winter, 2005). The last approach has the advantage that work can be defined separately at different joints, thus allowing investigation of the contribution of individual joints or body parts to the total mechanical work. Furthermore, it was found to be the most valid and discriminating approach in children with CP (Van de Walle et al., 2012).

For the examination of the gait pattern, the body is usually divided into two functional units: the locomotor unit and the passenger unit. The passenger unit consists of head, arms and trunk (HAT) and has minimal postural change in typical adult walking. Due to the high efficiency of typical gait the demands on the passenger unit are reduced to a minimum, despite its large and heavy mass of approximately $70 \%$ of the body weight (Perry \& Burnfield, 2010). In typical gait, arm swing has only minor effects on metabolic energy cost, but restriction of the arms leads to a less efficient gait pattern (Umberger, 2008).

In pathological gait, such as in $\mathrm{CP}$, the motions of the trunk as well as the arms often increase due to compensation strategies (Bruijn, Meyns, Jonkers, Desloovere, \& Duysens, 2011; Meyns et al., 2011; Molenaers et al., 2006; Romkes et al., 2007). These increased movements at the upper body can affect gait efficiency in different ways. In children with hemiplegia increased arm swing at the unaffected side seems to be aimed at controlling the total body angular momentum (Bruijn et al., 2011). As increased total body angular momentum has been linked to increased metabolic energy, the increased arm swing probably helps in maintaining gait efficiency (Ortega, Fehlman, \& Farley, 2008; Umberger, 2008). Still, increased movement at the trunk and pelvis can also lead to decreases in gait efficiency (Van de Walle et al., 2007). Until now, most evaluation of gait efficiency in children with CP did not focus on the HAT. However, children with diplegia often experience problems with trunk control and could benefit from evaluation of the HAT (Romkes et al., 2007; Woollacott \& Shumway-Cook, 2005). Investigation of mechanical work of the two decomposed units may contribute in the identification of sources of increased energy cost and improve insights in the role of the HAT in pathological gait. As energy expenditure changes as a function of age, it is important that changes in pathological paediatric gait are compared with age-related reference data (Van de Walle et al., 2010). To the best of our knowledge, so far, there is no information on mechanical work at the HAT in paediatric gait.

Therefore the purpose of this study was to investigate the contribution of the HAT to the total mechanical work in children with diplegia in comparison to age-related typical children. We hypothesized that mechanical energy estimation by integration of the joint powers can deliver valuable insights about the role of the passenger unit in decreased gait efficiency in children with diplegia.

\section{Methods}

\subsection{Participants}

The study population consisted of 25 ambulatory children with CP (child_DI) recruited from the clinical database of the University Hospital Pellenberg and 25 age-related children with typical development (child_TD) selected from a prior study (Van de Walle et al., 2010). Inclusion criteria were age between 5.5 and 12.5 years for all subjects, and spastic diplegic CP, Gross Motor Function Classification System (GMFCS) levels I-II for child_DI (Palisano et al., 1997). Exclusion criteria were any history of orthopaedic, neurological, cardiovascular pathologies or diagnosis of maturational problems for child_TD and walking aids, orthopaedic surgery within 2 years and Botulinum Toxin treatment within 4 months prior to the evaluation for child_DI. Furthermore, all subjects required the collection of three trials with valid bilateral kinetics as well as full visibility for total body kinematics during gait analysis. For child_DI 7 subjects had to be excluded after testing because no valid bilateral kinetics could be collected due to age-related or pathological short step length (age: $8.2 \pm 2.4$ years; 3 children with GMFCS-level I and 4 children with GMFCS-level II). Group characteristics and anthropometrics are shown in Table 1 . Clinical data for child_DI are shown in Table 2A. The study was approved by the local ethical committee and all subjects or their legal guardian provided written informed consent.

Table 1

Description of anthropometrics and walking speed (mean \pm SD) for children with diplegia and children with typical development. GMFCS: Gross Motor Function Classification System.

\begin{tabular}{lll}
\hline & Diplegia & Typical development \\
\hline Number & 18 & 25 \\
Gender (male/female) & $10 / 8$ & $11 / 14$ \\
Age (years) & $9.6 \pm 2.0$ & $9.3 \pm 2.0$ \\
Height (m) & $1.37 \pm 0.13$. & $140 \pm 0.13$ \\
Body mass (kg) & $30.1 \pm 8.5$ & $31.1 \pm 7.5$ \\
GMFCS (level I/II) & $11 / 7$ & - \\
\hline
\end{tabular}


Table 2

(A) Clinical data (spasticity, strength and selectivity) for children with diplegia (mean and standard deviation and [range]). (B) Spatio-temporal gait parameters and (C) trunk movement during walking for children with diplegia and children with typical development. For children with diplegia data are provided for the total group as well as for each GMFCS-level separately.

\begin{tabular}{|c|c|c|}
\hline & Diplegia (GMFCS-level I/II) & Typical development \\
\hline \multicolumn{3}{|c|}{ A. Clinical data } \\
\hline \multicolumn{3}{|c|}{ Spasticity (Modified Ashworth Scale) } \\
\hline Hipflexors & $0.7 \pm 0.5(0.5 \pm 0.6[0-1.5] / 1.0 \pm 0.3[1-1.5])$ & \\
\hline Hamstrings & $1.3 \pm 0.6(1.1 \pm 0.6[0-2] / 1.5 \pm 0.3[1-2])$ & \\
\hline Rectus femoris & $0.6 \pm 0.7(0.6 \pm 0.7[0-2] / 0.8 \pm 0.8[0-2])$ & \\
\hline Gastrocnemius & $1.5 \pm 0.7(1.4 \pm 0.8[0-3] / 1.8 \pm 0.6[1-3])$ & \\
\hline \multicolumn{3}{|c|}{ Strength (Manual Muscle Testing) } \\
\hline Abdominal muscles & $3.4 \pm 1.1(3.5 \pm 1.4[2-5] / 3.3 \pm 0.5[3-4])$ & \\
\hline Back extensors & $4.5 \pm 0.9(4.6 \pm 1.0[2-5] / 4.3 \pm 0.8[3-5])$ & \\
\hline Hip extensors & $3.9 \pm 0.5(4.0 \pm 0.6[3-5] / 3.7 \pm 0.3[3-4])$ & \\
\hline Hip flexors & $4.0 \pm 0.5(4.1 \pm 0.5[3-5] / 3.8 \pm 0.4[3-4])$ & \\
\hline Knee extensors & $4.1 \pm 0.5(4.2 \pm 0.5[3-5] / 3.9 \pm 0.2[3-4])$ & \\
\hline Knee flexors & $3.9 \pm 0.5(4.1 \pm 0.5[3-5] / 3.6 \pm 0.2[3-4])$ & \\
\hline Dorsiflexors & $3.8 \pm 0.5(4.1 \pm 0.4[3-5] / 3.5 \pm 0.5[2-4])$ & \\
\hline Plantar flexors & $3.8 \pm 0.5(3.9 \pm 0.5[3-5] / 3.5 \pm 0.5[2-5])$ & \\
\hline Selectivity (SMCT) & $3.6 \pm 0.5(3.7 \pm 0.4[3-4] / 3.4 \pm 0.7[2-4])$ & \\
\hline \multicolumn{3}{|l|}{ B. Spatio-temporal data } \\
\hline Walking speed (m/s) & $1.11 \pm 0.16(1.16 \pm 0.12 / 1.10 \pm 0.19)$ & $1.25 \pm 0.14$ \\
\hline Stride length $(\mathrm{m})$ & $1.04 \pm 0.14(1.09 \pm 0.09 / 0.96 \pm 0.18)$ & $1.15 \pm 0.11$ \\
\hline Stride time $(\mathrm{s})$ & $0.92 \pm 0.10(0.95 \pm 0.09 / 0.88 \pm 0.11)$ & $0.93 \pm 0.09$ \\
\hline Cadence (steps/min) & $133 \pm 15(128 \pm 13 / 141 \pm 15)$ & $130 \pm 13$ \\
\hline Percentage foot off (\%) & $60.0 \pm 2.1(59.6 \pm 2.0 / 60.6 \pm 2.0)$ & $57.7 \pm 1.9$ \\
\hline \multicolumn{3}{|c|}{ C. Trunk range of motion } \\
\hline Sagital plane $\left({ }^{\circ}\right)$ & $8.3 \pm 3.6(7.5 \pm 4.1 / 9.6 \pm 2.3)$ & $5.3 \pm 2.3$ \\
\hline Frontal plane $\left(^{\circ}\right)$ & $9.0 \pm 5.8(7.8 \pm 6.3 / 10.8 \pm 4.3)$ & $4.8 \pm 2.7$ \\
\hline Transverse plane $\left(^{\circ}\right)$ & $10.4 \pm 4.0(9.7 \pm 3.9 / 11.4 \pm 4.1)$ & $7.9 \pm 3.1$ \\
\hline
\end{tabular}

SMCT, Selective Motor Control Test; GMFCS, Gross Motor Function Classification System.

\subsection{Procedure}

During gait analysis, total body kinematics and kinetics were collected while walking on a 10-m walkway at self selected comfortable walking speed using an eight-camera VICON System with the Plug In Gait marker set (Mx camera-workstation, $100 \mathrm{~Hz}$, VICON, Oxford Metrics, Oxford, UK) and two embedded force plates in line, one after the other $(0.4 \mathrm{~m} \times 0.5 \mathrm{~m}$, 1500 Hz, Advanced Mechanical Technology Inc., Watertown, MA) (Van de Walle et al., 2010). For child_DI the gait analysis was part of a routine clinical evaluation and was therefore combined with a clinical examination of range of motion, alignment, spasticity and muscle strength and selectivity in order to have a complete clinical picture of the patients motor functioning. The mean of three trials per subject was used for data analysis.

\subsection{Calculations}

Mechanical work was estimated by the integrated joint power approach $\left(W_{\mathbf{j}}\right)$ (Van de Walle et al., 2012). $W_{\mathrm{j}}$ was obtained by separate integration of positive and negative net joint power profiles [ $\mathrm{J} \mathrm{kg}^{-1} \mathrm{~s}^{-1}$ ] for neck, left and right shoulder, left and right elbow, left and right wrist, waist, left and right hip, left and right knee and left and right ankle, as obtained from the Vicon Plug In Gait model (Davis, Ounpuu, Tyburski, \& Gage, 1991; Kadaba, Ramakrishnan, \& Wootten, 1990). Subsequently positive and negative work performed at each joint separately were summed for all joints [J kg $\left.\mathrm{kg}^{-1}\right]$. The sum of positive and negative net joint work of all joints resulted in respectively the total positive $\left(W_{\mathrm{j}}^{+}\right)$and negative net joint work ( $\left.W_{\mathrm{j}}^{-}\right)$ (Appendix, Eqs. (1) and (2)). To obtain total net joint work $\left(W_{\mathrm{j}}^{\text {tot }}\right)$ the sum of $W_{\mathrm{j}}^{+}$and $\left|W_{\mathrm{j}}^{+}\right|$was made (Appendix, Eq. (3)). $W_{\mathrm{j}}^{\text {tot }}$ was calculated for the total body with all 14 joints $\left(W_{\mathrm{j}}^{\text {tot }}{ }_{-} \mathrm{TB}\right)$ but also for the locomotor unit (work at the lower limbs, $W_{\mathrm{j}}^{\text {tot }}{ }_{-} \mathrm{LL}$, 6 joints) and for the passenger unit (work at the HAT, $W_{\mathrm{j}}^{\text {tot }}$ HAT, 8 joints) as well as for trunk ( $W_{\mathrm{j}}^{\text {tot }}$ trunk), arms $\left(W_{\mathrm{j}}^{\text {tot }} \_\right.$arms) and head ( $W_{\mathrm{j}}^{\text {tot }}$ head) separately.

Relative contribution of the HAT to the total mechanical work was calculated as $W_{\mathrm{j}}^{\text {tot }}{ }_{-} \mathrm{HAT} / W_{\mathrm{j}}^{\text {tot }}$-TB [\%].

Furthermore, relative contributions of head, arms and trunk to $W_{\mathrm{j}}^{\text {tot }}$ HAT were calculated. The changes in mechanical work in child_DI compared to child_TD were calculated for each parameter separately and expressed as a percentage.

\subsection{Statistical analysis}

Statistical analysis was performed with SPSS (IBM ${ }^{\circledR}$ SPSS ${ }^{\circledR}$ Statistics, Inc., version 19). Initial power calculations determined that minimum 17 children in each group were needed to detect a difference of two standard deviations with a 


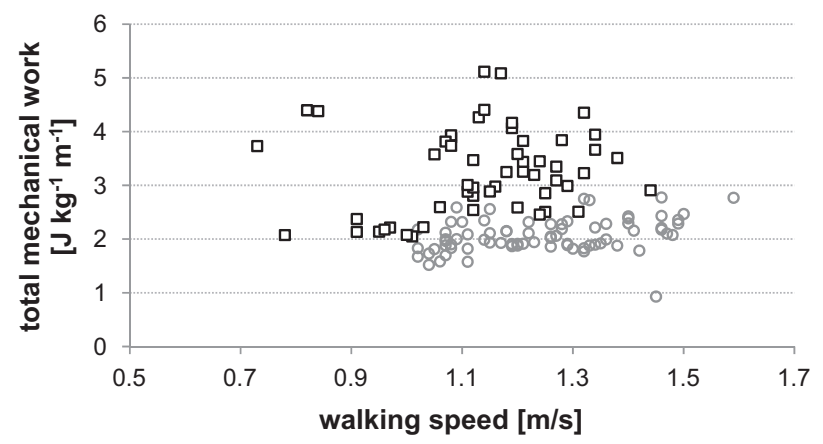

Fig. 1. Total mechanical work as a function of walking speed for children with typical development (grey dots) and children with diplegia (black squares).

power of 0.8. Normal distributions were confirmed by Kolmogorov-Smirnov test. Differences between child_DI and child_TD and between GMFCS-level within child_DI were investigated by independent samples $t$-test. Level of significance was set at $p<0.05$.

\section{Results}

\subsection{Changes in spatio-temporal data and trunk range of motion}

Spatio-temporal data are shown in Table 2B. The walking speed was significantly higher, stride length significantly lower and percentage foot off significantly later for child_TD than for child_DI $(p<0.001)$. No significant differences were found for stride time $(p=0.635)$ and cadence $(p=0.281)$. To further investigate the influence of walking speed, $W_{j}^{\text {tot }}$ TB of all trials separately for each subject were plotted against walking speed (Fig. 1). Although speed was significantly different between both groups, there is a clear overlap in walking speed. Trunk range of motion was significantly increased in child_DI in all planes $(p<0.001)$ (Table 2C).

\subsection{Changes in mechanical work in children with diplegia}

$W_{\mathrm{j}}^{\text {tot }}$ TB was significantly higher in child_DI compared to child_TD $(p<0.001)$ (Fig. 2A). The increase of $W_{\mathrm{j}}^{\text {tot }}$ TB in child_DI was caused by a small increase in $W_{j}^{\text {tot }}$ LL (37\%) and a large increase in $W_{j}^{\text {tot }}$ HAT (167\%) (Table 3). The increase of $W_{\mathrm{j}}^{\text {tot }}$ HAT was caused by an increase in net joint work at head, arms and trunk which were all significantly higher in child_DI compared to child_TD $(p<0.001)$ (Table 3$)$. The highest increase was seen for the arms (222\%) followed by the trunk $(199 \%)$. $W_{\mathrm{j}}^{\text {tot }}$ head was twice as high in child_CP compared to child_TD (114\%).

The relative contribution of the $W_{j}^{\text {tot }}$ HAT to $W_{j}^{\text {tot }}$ TB was significantly higher in child_DI $(22 \pm 7 \%)$ than in child_TD $(13 \pm 3 \%)(p<0.001)$. The relative contribution of the arms to $W_{\mathrm{j}}^{\text {tot }}$.HAT was small in both groups and not different between the two groups (Table 3). The contribution of the trunk to $W_{\mathrm{j}}^{\text {tot }}$ HAT was within the same range as that of the head. The contribution of the head was found to be significantly higher in child_DI than in child_TD; the contribution of the trunk was not different between both groups.

\subsection{Subgroup analysis by GMFCS-level}

No significant differences between $W_{\mathrm{j}}^{\text {tot }}$ TB in children with GMFCS I $\left(3.14 \pm 0.80 \mathrm{~J} \mathrm{~kg}^{-1} \mathrm{~m}^{-1}\right)$ compared to children with GMFCS II $\left(3.23 \pm 0.82 \mathrm{~J} \mathrm{~kg}^{-1} \mathrm{~m}^{-1}\right)$ were found $(p=0.83)$. Also no differences between the two groups were found for $W_{\mathrm{j}-L L}$ $(p=0.42)$. Absolute and relative work at the HAT were higher in children with GMFCS II $\left(0.90 \pm 0.35 \mathrm{~J} \mathrm{~kg}^{-1} \mathrm{~m}^{-1}, 27 \%\right)$ than in children with GMFCS I $\left(0.62 \pm 0.34 \mathrm{~J} \mathrm{~kg}^{-1} \mathrm{~m}^{-1}, 18 \%\right)$. Relative work was significantly higher $(p=0.01)$, the differences in absolute work were not significant $(p=0.13)$. The increase in $W_{\mathrm{j}}^{\text {tot }}$ HAT in children with GMFCS II was caused by a significant increase in work at the head $(p=0.01)$ and, the arms $(p=0.03)$. No significant increases in work at the trunk were found $(p=0.31)$ (Fig. 2B).

\section{Discussion}

In this study child_DI showed a decreased gait efficiency, as was previously demonstrated for both metabolic and mechanical energy expenditure (Bennett et al., 2005; Johnston, Moore, Quinn, \& Smith, 2004). The total mechanical work was further divided in work at the locomotor unit $\left(W_{\mathrm{j}}^{\text {tot }}-\mathrm{LL}\right)$ and work at the passenger unit $\left(W_{\mathrm{j}}^{\text {tot }}-\mathrm{HAT}\right)$ in order to obtain insights in the role of the passenger unit in pathological gait of children with diplegia. 

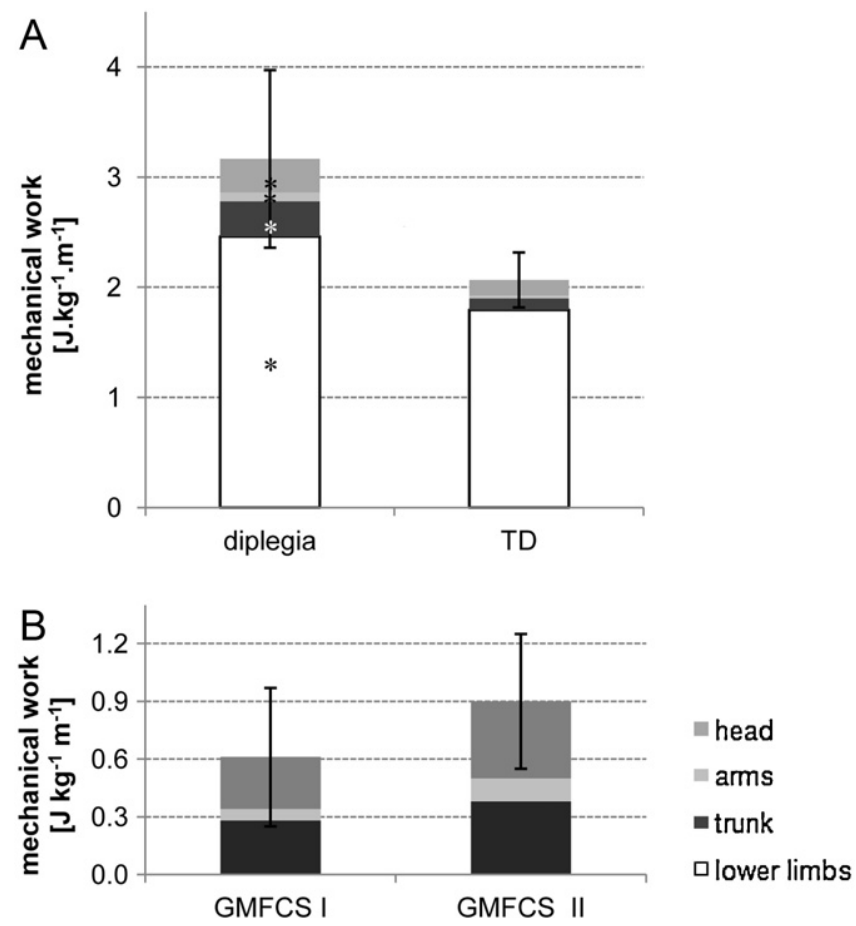

Fig. 2. (A) Mean total mechanical work divided in work at lower limbs, head, arms and trunk in children with diplegia and typical developing children (TD). (B) Comparison of mechanical work at head, arms and trunk for children with diplegia with GMFCS-levels I and II. Vertical bars represent standard deviation of the net joint work at the total body (A) and HAT (B).

\subsection{Work at the HAT}

In typical gait the mechanical work at the passenger unit was small compared to the total mechanical work (13\%), which confirms that the passenger unit has minimal energetic demands, despite its large mass (Perry \& Burnfield, 2010). In child_DI an increase of in $W_{j}^{\text {tot }}$ TB was found, which was caused by an increase of work at the locomotor unit (37\%) to a small extent, and by a remarkably larger increase in work at the passenger unit (167\%). Due to this disproportional increase, $W_{\mathrm{j}}^{\text {tot }}$ HAT accounted for $22 \%$ of $W_{j}^{\text {tot }}$ TB. Hence, in children with $\mathrm{CP}$, the demands on the passenger unit can no longer be considered minimal. Subgroup analysis by GMFCS-level indicated that in children with more severe gait disabilities the larger demands on the passenger unit become even more apparent. Increased work in children with diplegia was noted in all three components of the HAT.

Table 3

Differences in mechanical work (mean \pm SD) in children with diplegia and in children with typical development for total body ( $W_{j}^{\text {tot }}$ TB) and the different segments: locomotor unit $\left(W_{j}^{\text {tot }} \_\right.$LL) and passenger unit $\left(W_{j}^{\text {tot }}\right.$ HAT $)$ head $\left(W_{j}^{\text {tot }}\right.$ head $)$, arms $\left(W_{j}^{\text {tot }}\right.$ _arms $)$ and trunk $\left(W_{j}^{\text {tot }}\right.$ _trunk). For head, arms and trunk the relative contribution (percentage) to the work at the passenger unit is presented. For children with diplegia, the percentage increase compared to children with typical development is presented. Differences between both groups were investigated with independent-samples $t$-test.

\begin{tabular}{|c|c|c|c|c|}
\hline & $\begin{array}{c}\text { diplegia } \\
(n=18)\end{array}$ & $\begin{array}{l}\text { typical development } \\
(n=25)\end{array}$ & $p$-value & $\begin{array}{l}\text { increase in } \\
\text { diplegia }\end{array}$ \\
\hline & \multicolumn{2}{|c|}{$\mathrm{J} \mathrm{kg}^{-1} \mathbf{~ m}^{-1}$} & & $\%$ \\
\hline$W_{\mathrm{j}}^{\mathrm{tot}}-\mathrm{TB}$ & $3.18 \pm 0.78$ & $2.07 \pm 0.25$ & $<0.001$ & 54 \\
\hline $\mathrm{L}_{>} W_{\mathrm{j}}^{\text {tot }}{ }_{-} \mathrm{LL}$ & $2.45 \pm 0.48$ & $1.79 \pm 0.20$ & $<0.001$ & 37 \\
\hline$W_{\mathrm{j}}^{\text {tot }}{ }^{-} \mathrm{HAT}$ & $0.72 \pm 0.37$ & $0.27 \pm 0.09$ & $<0.001$ & 167 \\
\hline${ }^{L}>W_{j}^{\text {tot }}$ head & $0.32 \pm 0.16(44 \pm 7 \%)$ & $0.14 \pm 0.05(50 \pm 8 \%)$ & $<0.001(0.022)$ & 129 \\
\hline$W_{\mathrm{j}}^{\text {tot }}$ _arms & $0.08 \pm 0.05(12 \pm 4 \%)$ & $0.03 \pm 0.01(10 \pm 5 \%)$ & $<0.001(0.240)$ & 167 \\
\hline$W_{j}^{\text {tot }}{ }^{-}$trunk & $0.32 \pm 0.19(44 \pm 7 \%)$ & $0.11 \pm 0.04(40 \pm 7 \%)$ & $<0.001(0.091)$ & 190 \\
\hline
\end{tabular}




\subsection{Work at the arms}

The work at the arms was three times as high as in typical gait but as its contribution to the total mechanical work was only 3\%, the impact on overall gait efficiency remained limited. This might explain why experimental restraint of the arms had only small effects on metabolic energy expenditure (Umberger, 2008). The small contributions of the arms in both typical and pathological gait to $W_{\mathrm{j}}^{\text {tot }}$-TB can be explained by their relative small mass, as even with high increases in angular velocity, joint power remained small. Nevertheless, despite the relative small work at the arms, they may play an important role in maintaining balance and controlling angular momentum (Bruijn et al., 2011). The increases in work at the arms noted in children with diplegia are probably caused by increased arm movements to ensure postural stability. These small increases only have minor impact on the increase of $W_{\mathrm{j}}^{\text {tot }}$ TB. On the other hand, through controlling angular momentum they may prevent larger increases in work at trunk and lower limbs.

\subsection{Work at trunk and head}

The trunk and surprisingly also the head were the main contributors to the increased $W_{\mathrm{j}}^{\text {tot }}$ HAT in children with diplegia. The segmental mass of head and trunk is much larger than that of the arms and even small changes in angular velocity will result in an increase in muscle power and thus net joint work. $W_{j}^{\text {tot }}$ head was, despite its smaller mass, about as high as $W_{j}^{\text {tot }}$ trunk. This may be related to higher angular velocities. Cromwell et al. (2001) investigated the kinematics of the trunk, neck and head during walking and found higher excursions in neck and head compared to the trunk, which may lead to higher angular velocities. This hypothesis must be confirmed by further research that relates kinematic changes to changes in work of the passenger unit. The inability to maintain efficient movement of the passenger unit might be attributed to the passenger unit itself, but also to compensatory movements of the passenger unit as a reaction to deviations at the lower limbs. In the first case the increased work might be caused by a lack of muscle coordination and balance control at the HAT, which is known to be affected in children with diplegia (Romkes et al., 2007; Rosenbaum et al., 2007; Woollacott \& Shumway-Cook, 2005). In the second case, the increase in work at the HAT might be attributed to increased upper body movement to compensate for unstable gait, secondary to deviations at the lower limbs (Molenaers et al., 2006; Romkes et al., 2007). The increase in range of motion of the movements at the trunk in all three planes in child_DI compared to child_TD supports this hypothesis. Also, a combination of primary, at the passenger unit itself, and secondary problems (compensatory strategies) might be responsible for the increase of work at the HAT. However, this cannot be confirmed by the results of the current study. Therefore, a follow-up study will aim at in depth analysis of changes in total body kinematics in relation with changes in work.

\subsection{Subgroup analysis}

Subgroup analysis suggested that the work at the HAT was higher in children with GMFCS-level II compared to children with GMFCS-level I. Furthermore, the children with GMFCS-II were more affected at all clinical levels (spasticity, strength, selectivity and functional gait parameters) and showed higher movement of the trunk in all planes. This indicates that the role of the passenger unit in decreased gait efficiency becomes more apparent in children with more severe gait problems.

\subsection{Limitations of the study}

From a clinical point of view it is most interesting to assess mechanical cost at comfortable walking speed. This resulted in a significant different walking speed between child_DI and the typical controls that could have potentially influenced mechanical cost. As mechanical cost was higher in child_DI at all walking speeds, we believe that this was not the case in the current study.

The net joint work approach for mechanical energy estimation proved useful in gaining insights in the role of separate segments in increased mechanical cost of walking. This technique requires the collection of valid bilateral kinetics within one trial, which is known to be more difficult in younger children and in children with more severe gait pathologies (Schepens \& Detrembleur, 2009). Nevertheless, we were able to collect valid data for 18 out of the 25 children with CP. In our gait laboratory the force plates were placed in line, one after the other. A third force plate in a row, next to the force plates in line, would allow the collection of valid kinetics in children with short step length and a wider base of support.

The subgroup analysis was based on a small sample size. The indications from this analysis could therefore not be supported by significant changes. An increase in sample sizes within each GMFCS-level would enhance the generalizability of the results.

\subsection{Clinical implications}

Children with CP present with decreased gait efficiency. In order to direct treatment to avenues that can improve gait efficiency it is important to identify the causes of decreased gait efficiency. This requires techniques that allow decomposition of total energy expenditure into separate segments. The use of net joint work allowed to detect and increase in total work and to look at the contribution of the separate segments at the same time. The results from this study indicate 
that the passenger unit plays an important role in the decreased gait efficiency. The estimation of mechanical energy of the HAT appeared invaluable as it can help to identify the sources of increased work during clinical evaluation of functional children with diplegia.

\section{Conclusion}

In conclusion, the results of this study highlighted the substantial contribution of the passenger unit in decreased gait efficiency in children with spastic diplegia.

\section{Acknowledgements}

This study was supported by grant G827 from the Artesis University College of Antwerp. The authors like to thank the colleagues of the Clinical Motion Analysis Laboratory of the University Hospital Pellenberg for their contribution to the data collection and all the children for their willingness to participate.

\section{Appendix A. Work calculated by integration of joint powers}

$$
\begin{aligned}
& W_{\mathrm{j}}^{+}=\sum_{i=1}^{N}\left(\int P_{\text {joint }} d t\right), \text { if } \int P_{\text {joint }}>0 \\
& W_{\mathrm{j}}^{-}=\sum_{i=1}^{N}\left(\int P_{\text {joint }} d t\right), \text { if } \int P_{\text {joint }}<0 \\
& W_{\mathrm{j}}^{\text {tot }}=W_{\mathrm{j}}^{+}+\left|W_{\mathrm{j}}^{-}\right|
\end{aligned}
$$

$N$ is the number of joints, 14 in case of the total body, 6 in case of the locomotor unit and 8 in case of the passenger unit

\section{References}

Bennett, B. C., Abel, M. F., Wolovick, A., Franklin, T., Allaire, P. E., \& Kerrigan, D. C. (2005). Center of mass movement and energy transfer during walking in children with cerebral palsy. Archives of Physical Medicine $\mathcal{E}$ Rehabilitation, 86, 2189-2194.

Bruijn, S. M., Meyns, P., Jonkers, I., Desloovere, K., \& Duysens, J. (2011). Control of angular momentum during walking in children with cerebral palsy. Research in Developmental Disabilities, 32, 2860-2866.

Cromwell, R. L., Aadland-Monahan, T. K., Nelson, A. T., Stern-Sylvestre, S. M., \& Seder, B. (2001). Sagittal plane analysis of head, neck, and trunk kinematics and electromyographic activity during locomotion. The Journal of Orthopaedic and Sports Physical Therapy, 31, $255-262$.

Davis, R., Ounpuu, S., Tyburski, D., \& Gage, J. R. (1991). A gait analysis data collection and reduction technique. Human Movement Sciences, $10,575-587$.

Gage, J. R. (1991). Gait analysis in cerebral palsy. London: McKeith Press. pp. 61-95.

Johnston, T. E., Moore, S. E., Quinn, L. T., \& Smith, B. T. (2004). Energy cost of walking in children with cerebral palsy: Relation to the Gross Motor Function Classification System. Developmental Medicine E Child Neurology, 46, 34-38.

Kadaba, M. P., Ramakrishnan, H. K., \& Wootten, M. E. (1990). Measurement of lower extremity kinematics during level walking. Journal of Orthopaedic Research, 8, 383-392.

Kerr, C., Parkes, J., Stevenson, M., Cosgrove, A. P., \& McDowell, B. C. (2008). Energy efficiency in gait, activity, participation, and health status in children with cerebral palsy. Developmental Medicine \& Child Neurology, 50, 204-210.

Meyns, P., Van Gestel, L., Massaad, F., Desloovere, K., Molenaers, G., \& Duysens, J. (2011). Arm swing during walking at different speeds in children with Cerebral Palsy and typically developing children. Research in Developmental Disabilities, 32, 1957-1964.

Molenaers, G., Desloovere, K., Van Campenhout, A., Pauwels, P., Ortibus, E., \& Van de Walle, P. (2006). Effect of ankel foot orthoses on 3D trunk and pelvis motion during gait in children with CP. 1st joint ESMAC-GCMAS meeting (Vol. 24, supplement 2, pp. s174-s175).

Ortega, J. D., Fehlman, L. A., \& Farley, C. T. (2008). Effects of aging and arm swing on the metabolic cost of stability in human walking. Journal of Biomechanics, 41, 3303-3308.

Palisano, R., Rosenbaum, P., Walter, S., Russell, D., Wood, E., \& Galuppi, B. (1997). Development and reliability of a system to classify gross motor function in children with cerebral palsy. Developmental Medicine E Child Neurology, 39, 214-223.

Perry, J., \& Burnfield, J. (2010). Basic functions. In J. Perry \& J. Burnfield (Eds.), Gait analysis: Normal and pathological function (2nd ed., pp. 19-48). Thorofare, USA: Slack Incorporated.

Piccinini, L., Cimolin, V., Galli, M., Berti, M., Crivellini, M., \& Turconi, A. C. (2007). Quantification of energy expenditure during gait in children affected by cerebral palsy. Europa Medicophysica, 43, 7-12.

Romkes, J., Peeters, W., Oosterom, A. M., Molenaar, S., Bakels, I., \& Brunner, R. (2007). Evaluating upper body movements during gait in healthy children and children with diplegic cerebral palsy. Journal of Pediatric Orthopedics B, 16, 175-180.

Rosenbaum, P., Paneth, N., Leviton, A., Goldstein, M., Bax, M., Damiano, D., et al. (2007). A report: The definition and classification of cerebral palsy April 2006. Developmental Medicine \& Child Neurology, 109, 8-14.

Schepens, B., \& Detrembleur, C. (2009). Calculation of the external work done during walking in very young children. European Journal of Applied Physiology, 107, 367-373.

Umberger, B. R. (2008). Effects of suppressing arm swing on kinematics, kinetics, and energetics of human walking. Journal of Biomechanics, 41, 2575-2580.

Van de Walle, P., Desloovere, K., Truijen, S., Gosselink, R., Aerts, P., \& Hallemans, A. (2010). Age-related changes in mechanical and metabolic energy during typical gait. Gait E' Posture, 31, 495-501.

Van de Walle, P., Gosselink, R., Desloovere, K., Molenaers, G., Hallemans, A., Op de Beeck, N., et al. (2007). Effect of trunk and pelvic motion on gait efficiency. 16th Annual Meeting of ESMAC. 
Van de Walle, P., Hallemans, A., Schwartz, M., Truijen, S., Gosselink, R., \& Desloovere, K. (2012). Mechanical energy estimation during walking: Validity and sensitivity in typical gait and in children with cerebral palsy. Gait \& Posture, 35, 231-237.

van den Hecke, A., Malghem, C., Renders, A., Detrembleur, C., Palumbo, S., \& Lejeune, T. M. (2007). Mechanical work, energetic cost, and gait efficiency in children with cerebral palsy. Journal of Pediatric Orthopedics, 27, 643-647.

Winter, D. A. (2005). Biomechanics and motor control of human movement (3rd ed.). Hoboken, NJ: John Wiley and Sons Inc.

Woollacott, M. H., \& Shumway-Cook, A. (2005). Postural dysfunction during standing and walking in children with cerebral palsy: What are the underlying problems and what new therapies might improve balance? Neural Plasticity, 12, 211-219, (Discussion 263-272). 